\title{
UTILIZAÇÃO DE PLANEJAMENTO FATORIAL NO PREPARO DE AMOSTRAS DE DETERGENTE EM PÓ PARA A DETERMINAÇÃO DE FÓSFORO POR ANÁLISE EM FLUXO
}

\author{
Fabíola Soraia Vital Campos Barbosa da Silva \\ Departamento de Química Fundamental, Universidade Federal de Pernambuco, Av. Prof. Luiz Freire, s/n, 50740-550 Recife-PE/Instituto \\ Federal de Educação, Ciência e Tecnologia de Pernambuco - Campus Recife, Av. Prof. Luiz Freire, 500, 50740-540 Recife - PE, Brasil \\ Valdinete Lins da Silva \\ Departamento de Engenharia Química, Universidade Federal de Pernambuco. Av. Prof. Artur de Sá, s/n, 50740-521 Recife - PE, Brasil \\ André Fernando Lavorante \\ Departamento de Química, Universidade Federal Rural de Pernambuco, Rua Dom Manoel de Medeiros, s/n, 52171-900 Recife - PE, Brasil \\ Ana Paula Silveira Paim* \\ Departamento de Química Fundamental, Universidade Federal de Pernambuco, Av. Prof. Luiz Freire, s/n, 50740-550 Recife - PE, Brasil
}

Recebido em 31/8/09; aceito em 7/1/10; publicado na web em 23/4/10

\begin{abstract}
EVALUATION OF SAMPLE PREPARATION OF THE DETERGENT POWDER USING FACTORIAL DESIGN. The decomposition of detergent powder samples in a microwave oven and autoclave was evaluated. To establish the best experimental conditions a $2^{5}$ factorial design was performed, varying the conditions in autoclave and microwave digestion and flow system parameters for the determination of phosphorus. The best composition was: $0.2 \mathrm{~mL}$ sulfuric acid; $500 \mathrm{~W}$ power and a 2 min time interval; $6 \mathrm{mmol} \mathrm{L}^{-1}$ of ascorbic acid and $16 \mathrm{mmol} \mathrm{L}^{-1}$ of molybdate to flow system. This factor levels use less reagents than the reference method. No statistically significant differences were found between the autoclave and microwave oven responses at the $95 \%$ confidence level.
\end{abstract}

Keywords: digestion; phosphate; detergents.

\section{INTRODUÇÃO}

O fósforo se apresenta em diferentes formas químicas, fósforo inorgânico (ortofosfato e polifosfato) e fósforo orgânico (combinado com a matéria orgânica). No meio ambiente, quando em excesso, é um dos responsáveis pela eutrofização de lagos e rios, ou seja, provoca o enriquecimento da água com nutrientes que favorecem a proliferação de algas tóxicas. ${ }^{1,2}$ Algas em grande quantidade podem levar ao excesso de matéria orgânica na água que, quando clorada, formará produtos cancerígenos como, por exemplo, os organoclorados. ${ }^{3}$

O descarte dos compostos de fósforo nos corpos hídricos está diretamente ligado ao uso de detergentes em pó, cuja fonte é o tripolifosfato de sódio ( $\mathrm{STPP}, \mathrm{Na}_{5} \mathrm{P}_{3} \mathrm{O}_{10}$ ). Os fosfatos inorgânicos são importantes na composição dos detergentes, visto que suas reações com certos íons de metais, como cálcio e magnésio, diminuem a dureza da água. ${ }^{4}$

Há muitos anos os detergentes em pó, que são consumidos em grande parte da Europa Ocidental e em regiões industrializadas como América do Norte, Japão e Coréia do Sul, não contêm STPP. Na China, muitas regiões têm banido o uso de fosfato. Porém, os fosfatos permanecem como principal agente estruturante em detergentes de alta performance e ainda são encontrados nas Américas do Sul e Central, África, Leste Europeu e Ásia. ${ }^{5}$

Para se ter ideia do impacto do uso de detergentes em pó à base de STPP, a concentração do fósforo em águas residuárias de lavanderia pode ser até 9 vezes maior que o permitido na legislação e o consumo de água no processo total pode chegar a $5 \mathrm{~L} \mathrm{~kg}^{-1}$ de roupa lavada. ${ }^{6}$

A resolução CONAMA $359 / 05^{7}$ dispõe sobre a regulamentação do teor de fósforo em detergentes em pó para o uso no mercado nacional, visando a redução e eventual eliminação do aporte de fósforo nos corpos de água. Com isso, torna-se importante determinar a sua concentração em amostras de detergentes em pó, visto que estes produtos fazem parte do esgoto doméstico.

*e-mail: anaspaim@ufpe.br
Para determinar a concentração de fósforo em detergente em pó, dependendo da técnica de detecção utilizada, é necessário que o fósforo seja convertido à forma de ortofosfato durante o preparo da amostra.

A radiação micro-ondas tem sido utilizada na etapa de preparo de amostras de diferentes matrizes. Os procedimentos de digestão podem empregar frascos abertos ou fechados ${ }^{8-11}$ e podem ser utilizados em batelada ou com sistemas de fluxo acoplados. ${ }^{12,13}$ Essa técnica apresenta como vantagens alta eficiência no aquecimento da mistura reacional, sem alterar a estrutura dos íons e/ou moléculas e reduzido tempo da digestão causado pelo controle da potência do forno. ${ }^{12}$

Diferentes procedimentos de preparo têm sido realizados em amostras para determinação de fósforo, os quais envolvem autoclave, forno de micro-ondas doméstico e comercial, mufla, biodigestor e termoreator. ${ }^{14-16}$ Entretanto, verifica-se na literatura que a maior parte dos procedimentos para determinação de fósforo são aplicados às águas, sendo que poucos são os trabalhos que analisam detergentes em pó, visto que se trata de uma matriz complexa que contém mais de 25 ingredientes diferentes. Basicamente, os componentes podem ser classificados em quatro grandes grupos: surfactantes, estruturantes, agentes de branqueamento e agentes auxiliares. O STPP faz parte do grupo dos estruturantes. ${ }^{5}$

Al-Tamrah e Osibanjo ${ }^{4}$ determinaram a concentração de ortofosfato em detergentes utilizando uma técnica quimioluminescente na qual as emissões moleculares são geradas dentro de uma cavidade que é introduzida em uma chama de difusão de hidrogênio. O método proposto mostrou-se preciso e razoavelmente rápido, não necessitando de uma pré-hidrólise da amostra.

Khanmohammadi e colaboradores ${ }^{5}$ propuseram um método para determinação simultânea de tripolifosfato de sódio (STPP), sulfato de sódio e alquilbenzenossulfonato linear em detergente em pó utilizando espectroscopia de infravermelho com transformada de Fourier com reflectância total atenuada. Neste método não foi necessário um pré-tratamento das amostras, apenas a sua dissolução em um solvente que não fosse reagir com o detergente e que também não interferisse 
na região espectral do analito. Entretanto, trata-se de um método que requer equipamento de elevado custo quando comparado com os métodos espectrofotométricos.

O procedimento clássico para a determinação de fósforo em detergente em pó consiste em um método gravimétrico. Os métodos gravimétricos ${ }^{17}$ apresentam a vantagem de serem diretos, no entanto, possuem como desvantagens o fato de serem mais susceptíveis a erros, mais demorados e pouco sensíveis. Métodos diretos são uma boa estratégia para a química analítica moderna, uma vez que buscam o processamento rápido das informações associado a procedimentos simples e confiáveis. ${ }^{18}$

O sistema de análise por injeção em fluxo (FIA) é considerado uma poderosa ferramenta para análise de rotina em larga escala, apresentando baixo custo operacional e elevada frequência de amostragem. ${ }^{19}$ Diversos conceitos inerentes a este processo continuam aparecendo na literatura entre eles, análise por injeção sequencial $(\mathrm{SIA})^{20}$ e multicomutação em fluxo. ${ }^{21}$

Um sistema de análise por injeção em fluxo (FIA) foi proposto para determinação de ortofosfato e fosfatos inorgânicos totais em detergentes. ${ }^{22}$ Neste sistema, a digestão foi realizada em linha utilizando-se um aquecedor elétrico e, em seguida, a solução foi resfriada e passava por um desborbulhador. Após este procedimento foram adicionados os reagentes e a mistura seguiu para o detector. As amostras de detergentes foram analisadas e, comparando-se o método proposto com o método de referência, foi verificado que os erros para o teor de $\mathrm{P}_{2} \mathrm{O}_{5}$ foram relativamente elevados, enquanto que para o teor de ortofosfato foi aceitável.

O planejamento fatorial ${ }^{23,24}$ é uma ferramenta estatística importante e simples. A observação dos efeitos das variáveis e de suas interações é de extrema importância para entender os processos que estão sendo monitorados em um determinado sistema. ${ }^{25}$

A aplicação do planejamento fatorial, tanto na parte de preparo da amostra quanto na escolha da quantidade de reagentes utilizada na quantificação, contribui para que se possa varrer adequadamente o espaço amostral, na busca das melhores condições de trabalho, bem como tirar informações sobre as variáveis que afetam o sistema, reduzindo o trabalho laboratorial. ${ }^{10}$

Neste trabalho foram avaliadas duas metodologias de digestão de amostras de detergentes em pó: autoclave e forno de micro-ondas com cavidade (Provecto Analítica, modelo DGT 100 Plus). Buscando estabelecer as melhores condições experimentais foram realizados três planejamentos fatoriais, nos quais se variaram as condições da digestão em autoclave, da digestão em micro-ondas e dos parâmetros do sistema em fluxo para determinação de fósforo. Após estabelecer as melhores condições experimentais, determinou-se a concentração de fósforo em detergentes em pó de larga comercialização e de diferentes fabricantes a fim de verificar se as mesmas estão atendendo aos valores especificados na legislação CONAMA 359/05. ${ }^{7}$

\section{PARTE EXPERIMENTAL}

\section{Reagentes e soluções}

Todas as soluções foram preparadas com água destilada e deionizada e todos os reagentes utilizados foram de grau analítico.

A solução estoque do padrão de $8 \mathrm{mg} \mathrm{L}^{-1}$ de fósforo foi obtida pesando-se 31,66 mg de tripolifosfato de sódio (doado pela ASA) e dissolvendo-se em $1000 \mathrm{~mL}$ de água.

A solução $340 \mathrm{mmol} \mathrm{L}-1$ ácido ascórbico $\left(\mathrm{C}_{6} \mathrm{H}_{8} \mathrm{O}_{6}\right)$ (Merck) foi preparada pesando-se $6 \mathrm{~g}$ de ácido ascórbico, dissolvendo-se em $100 \mathrm{~mL}$ de água destilada. Para o preparo da solução $60 \mathrm{mmol} \mathrm{L}^{-1}$ realizou-se a diluição adequada.

A solução $5,4 \mathrm{~mol} \mathrm{~L}^{-1}$ ácido sulfúrico foi preparada transfe- rindo-se uma alíquota de $30 \mathrm{~mL}$ de $\mathrm{H}_{2} \mathrm{SO}_{4}$ concentrado (Merck) em $100 \mathrm{~mL}$ de água.

Para o preparo da solução estoque de $32 \mathrm{mmol} \mathrm{L}^{-1}$ molibdato de amônio (QM), pesou-se $20 \mathrm{~g}$ do sal e dissolveu-se em $400 \mathrm{~mL}$ de água destilada. Após manter a solução sob agitação durante 4 h, transferiu-se para um balão volumétrico de $500 \mathrm{~mL}$ e completou-se o volume com água destilada. A solução $16 \mathrm{mmol} \mathrm{L}^{-1}$ foi preparada após a diluição adequada da solução estoque.

Para o preparo da solução estoque $9 \mathrm{mmol} \mathrm{L}^{-1}$ tartarato de potássio e antimônio (Vetec), pesou-se 3,0 g do tartarato de potássio e antimônio e dissolveu-se em um béquer de $250 \mathrm{~mL}$ com água destilada e, em seguida, transferiu-se para um balão volumétrico de 1000 mL e completou-se o volume com água destilada. Esta solução foi armazenada em frasco âmbar sob refrigeração.

Para compor a solução reagente, misturou-se em um béquer de 1000 $\mathrm{mL}, 35 \mathrm{~mL}$ de ácido sulfúrico concentrado em $500 \mathrm{~mL}$ de água destilada, em seguida adicionou-se $215 \mathrm{~mL}$ da solução estoque de $32 \mathrm{mmol} \mathrm{L}^{-1}$ molibdato de amônio e $72 \mathrm{~mL}$ da solução $9 \mathrm{mmol} \mathrm{L}^{-1}$ tartarato de potássio e antimônio. Posteriormente transferiu-se a mistura para um balão volumétrico de $1000 \mathrm{~mL}$ e completou-se o volume com água destilada.

\section{Amostras}

As amostras de detergente em pó foram adquiridas no comércio de Recife. As amostras (500 g) foram homogeneizadas e passaram por um processo de quarteamento (em um quarteador tipo Jones). Em seguida uma porção da amostra foi seca e moída em almofariz com pistilo para uniformizar as diferentes granulações. Em seguida foram pesadas e dissolvidas com água destilada.

\section{Planejamento experimental para o preparo da amostra em autoclave e forno de micro-ondas}

Para avaliar o processo de preparo de amostra de detergente em pó, foram comparados dois procedimentos de digestão: em autoclave (autoclave vertical Proenix, modelo AV - 75) e em forno de micro-ondas com cavidade (Provecto Analítica, modelo DGT 100 Plus). Inicialmente, trabalhou-se com soluções de STPP de concentração conhecida.

$\mathrm{Na}$ avaliação da determinação de fósforo em sistema de análise em fluxo com o preparo de amostra envolvendo autoclave, os fatores estudados foram o volume do ácido sulfúrico $(\mathrm{S})$, a concentração da solução de molibdato de amônio (M) e da solução de ácido ascórbico (A). A concentração analisada foi de $8 \mathrm{mg} \mathrm{L}^{-1}$ de fosfato.

Para isso, foi delineado e executado um planejamento fatorial $2^{3}$ completo acrescido de 7 repetições no ponto central, totalizando 15 ensaios. Os níveis superior e inferior dos fatores estudados foram, respectivamente, volume do ácido sulfúrico (S) 0,6 e 0,2 mL, concentração da solução de molibdato de amônio (M) 32 e $16 \mathrm{mmol} \mathrm{L}^{-1}$ e da solução de ácido ascórbico (A) 340 e 60 mmol L-1. Utilizou-se como resposta o retorno percentual, que é definido como a razão entre a concentração encontrada e a concentração da solução preparada multiplicada por 100 .

Alíquotas de $10 \mathrm{~mL}$ de cada solução de $8 \mathrm{mg} \mathrm{L}^{-1}$ de fosfato (na forma de STPP) foram transferidas para tubos de vidro rosqueados com capacidade interna de $20 \mathrm{~mL}$ e, em seguida, foi adicionado a cada tubo o volume adequado do ácido sulfúrico 5,4 $\mathrm{mol} \mathrm{L}^{-1}$.

Os tubos foram devidamente fechados e colocados em uma autoclave com pressão interna de $1 \mathrm{kgf} \mathrm{cm}^{-2}$ durante o intervalo de tempo de $1 \mathrm{~h}$.

$\mathrm{Na}$ avaliação da eficiência do preparo das amostras em forno de micro-ondas, manteve-se a temperatura em $120^{\circ} \mathrm{C}$ e foram estudados os seguintes fatores: o volume da solução de $\mathrm{H}_{2} \mathrm{SO}_{4}(\mathrm{~S})$, a concen- 
tração de molibdato de amônio (M), a solução de ácido ascórbico (A), o tempo de irradiação (t) e a potência do forno (W). Para tanto, foi realizado um planejamento fatorial $2^{5}$ completo, sem repetições, totalizando 32 experimentos, os quais foram parcialmente aleatórios. Os níveis superior e inferior dos fatores estudados foram, respectivamente, volume da solução de $\mathrm{H}_{2} \mathrm{SO}_{4}(\mathrm{~S})$ 0,6 e $0,2 \mathrm{~mL}$, concentração de molibdato de amônio (M) 32 e $16 \mathrm{mmol} \mathrm{L}^{-1}$, concentração de ácido ascórbico (A) 340 e 60 mmol L ${ }^{-1}$, tempo de irradiação (t) 6 e 2 min e potência do forno (W) 500 e $300 \mathrm{~W}$.

Em um frasco reacional do forno de micro-ondas, adicionou-se $10 \mathrm{~mL}$ da solução de $8 \mathrm{mg} \mathrm{L}^{-1}$ de fosfato e volume adequado do ácido sulfúrico $5,4 \mathrm{~mol} \mathrm{~L}^{-1}$. O frasco reacional foi colocado no forno de microondas, operado de acordo com as especificações de cada experimento.

Após serem digeridos, os padrões de fosfato foram analisados em um sistema de análise por injeção em fluxo com detecção a 880 $\mathrm{nm} .{ }^{26} \mathrm{Na}$ avaliação da melhor condição de preparo da amostra em forno de micro-ondas, conforme definido anteriormente, utilizou-se como resposta o retorno percentual.

\section{Teste de adição e recuperação}

Foram digeridas cinco amostras de detergente em pó, em duplicata. Em 10 tubos de vidro rosqueados, adicionou-se cerca de $0,1 \mathrm{~g}$ da amostra do detergente em pó, $10 \mathrm{~mL}$ de água destilada e $0,2 \mathrm{~mL}$ de $\mathrm{H}_{2} \mathrm{SO}_{4} 5,4 \mathrm{~mol} \mathrm{~L}^{-1}$. Em 5 tubos foi realizada a adição de $1 \mathrm{mg} \mathrm{L}^{-1} \mathrm{de}$ $\mathrm{P}$, nos outros 5 tubos não foi adicionado padrão. Para que as amostras fossem digeridas, os tubos foram fechados e colocados em autoclave com pressão interna de $1 \mathrm{kgf} \mathrm{cm}^{-2}$ durante o intervalo de tempo de $1 \mathrm{~h}$.

\section{Determinação da concentração de fosfato em amostras de detergentes utilizando sistema em fluxo e detecção espectrofotométrica}

Foram realizadas duas formas de abertura para cada amostra: uma utilizando a autoclave e outra, o forno de micro-ondas. Os experimentos foram realizados nas condições mais eficientes após avaliação dos resultados dos planejamentos.

Pesou-se $0,1 \mathrm{~g}$ da amostra do detergente em pó ${ }^{22}$ e transferiu-se para um tubo de vidro rosqueado (autoclave) ou frasco reacional (forno de micro-ondas), em seguida, adicionou-se $10 \mathrm{~mL}$ de água destilada e 0,2 $\mathrm{mL}$ do ácido sulfúrico $5,4 \mathrm{~mol} \cdot \mathrm{L}^{-1}$. A autoclave foi mantida à pressão interna de $1 \mathrm{kgf} \mathrm{cm}^{-2}$ durante o intervalo de tempo de $1 \mathrm{~h}$ e o forno de micro-ondas foi operado a uma potência de $500 \mathrm{~W}$ por 6 min.

As amostras de detergente após serem digeridas foram analisadas no sistema FIA. ${ }^{26}$ Foi possível comparar, através destes resultados, se o teor de fósforo nestes produtos está de acordo com os padrões estabelecidos pela legislação em vigor.

\section{RESULTADOS E DISCUSSÃO}

\section{Planejamento fatorial para avaliação das condições do preparo} da amostra em autoclave e forno de micro-ondas

No procedimento de preparo de amostras através de metodologia padrão ${ }^{27}$ são utilizados ácido sulfúrico e persulfato de sódio. Previamente foi realizada uma avaliação, verificando-se que a presença de persulfato não influenciava no preparo da amostra de detergente em pó, visto que este serve para converter fósforos orgânicos em fosfato. Por este motivo, foi realizado um planejamento fatorial completo $2^{3}$, utilizando a autoclave, para estudar os fatores que influenciam no preparo de amostra (volume do ácido sulfúrico, S) e no sistema de análise em fluxo (concentração de ácido ascórbico, A e de molibdato de amônio, M). Os valores encontrados estão mostrados na Tabela 1.
Tabela 1. Matriz de planejamento com os valores das respostas para a digestão em autoclave

\begin{tabular}{ccccc}
\hline Experimento & A & M & S & Resposta (\%) \\
\hline 1 & - & - & - & 89,8 \\
2 & + & - & - & 91,4 \\
3 & - & + & - & 94,9 \\
4 & + & + & - & 87,1 \\
5 & - & - & + & 88,5 \\
6 & + & - & + & 79,1 \\
7 & - & + & + & 85,3 \\
8 & + & + & + & 85,8 \\
9 & 0 & 0 & 0 & 84,7 \\
10 & 0 & 0 & 0 & 84,8 \\
11 & 0 & 0 & 0 & 82,5 \\
12 & 0 & 0 & 0 & 84,1 \\
13 & 0 & 0 & 0 & 83,7 \\
14 & 0 & 0 & 0 & 87,1 \\
15 & 0 & 0 & 0 & 82,0 \\
\hline
\end{tabular}

Empregando-se o nível de $95 \%$ de confiança, são considerados significativos o efeito principal A, S e o efeito de interação das três variáveis estudadas ASM, como pode ser observado na Tabela 2

Tabela 2. Efeitos principais e de interações entre as variáveis para a digestão em autoclave

\begin{tabular}{cc}
\hline & Efeitos \pm erro padrão \\
\hline Média & $87,7 \pm 0,6$ \\
A & $-3,8 \pm 1,2$ \\
M & $1,1 \pm 1,2$ \\
S & $-6,1 \pm 1,2$ \\
A x M & $0,1 \pm 1,2$ \\
A x S & $-0,7 \pm 1,2$ \\
M x S & $0,7 \pm 1,2$ \\
A x M x S & $4,8 \pm 1,2$ \\
\hline
\end{tabular}

Intervalo com $95 \%$ de confiança: $\mathrm{t}_{(6,0,95)} \times \mathrm{s}=1,2 \times 2,447=2,94$

A interação significativa entre os três fatores caracteriza um sistema cuja otimização deve ser realizada de forma multivariada. As três melhores condições em termos de resposta foram os experimentos 1 , 2 e 3. A condição mais econômica, entretanto, é a do experimento 1 , que corresponde a $60 \mathrm{mmol} \mathrm{L}^{-1}$ de ácido ascórbico (A), $16 \mathrm{mmol} \mathrm{L}^{-1}$ de molibdato (M) e 0,2 mL de ácido sulfúrico (S). Estes valores foram selecionados, pois também proporcionam menor consumo de reagentes quando comparados com o método padrão que utiliza 340 e $32 \mathrm{mmol}$ $\mathrm{L}^{-1}$ de ácido ascórbico e molibdato de amônio, respectivamente.

$\mathrm{Na}$ avaliação da melhor condição de preparo da amostra em forno de micro-ondas foi selecionado o retorno percentual como o parâmetro indicativo da condição em que houve melhor eficiência na digestão do analito.

Como não foram realizadas repetições, a significância dos efeitos foi estimada através da soma quadrática residual, considerando-se que efeitos de interação de ordem superior a três não são significativos. Os resultados não mostraram efeitos significativos (principal e de interação) para a variável tempo. Um gráfico normal também foi construído, chegando-se à mesma conclusão. Esta variável foi então excluída do planejamento, tornando-o um $2^{4}$ com repetições (Tabela 3 ).

Os novos cálculos realizados estão apresentados na Tabela 4. No nível de $95 \%$ de confiança, são significativos os efeitos principais, em $\mathrm{mg} \mathrm{L}^{-1}$ : M $(-5,6), \mathrm{S}(-4,9)$ e a interação A com M $(-6,9)$. Este efeito de interação também mostra a necessidade da otimização multivariada. 
Tabela 3. Matriz de planejamento com os valores das respostas para a digestão em forno de micro-ondas

\begin{tabular}{cccccc}
\hline Experimento & $\mathrm{M}$ & $\mathrm{A}$ & $\mathrm{S}$ & $\mathrm{W}$ & Resposta média (\%) \\
\hline 1 & -1 & -1 & -1 & -1 & 91,7 \\
2 & 1 & -1 & -1 & -1 & 91,2 \\
3 & -1 & 1 & -1 & -1 & 93,5 \\
4 & 1 & 1 & -1 & -1 & 79,4 \\
5 & -1 & -1 & 1 & -1 & 80,9 \\
6 & 1 & -1 & 1 & -1 & 86,2 \\
7 & -1 & 1 & 1 & -1 & 92,4 \\
8 & 1 & 1 & 1 & -1 & 77,6 \\
9 & -1 & -1 & -1 & 1 & 96,6 \\
10 & 1 & -1 & -1 & 1 & 93,7 \\
11 & -1 & 1 & -1 & 1 & 95,5 \\
12 & 1 & 1 & -1 & 1 & 86,8 \\
13 & -1 & -1 & 1 & 1 & 87,7 \\
14 & 1 & -1 & 1 & 1 & 90,1 \\
15 & -1 & 1 & 1 & 1 & 93,8 \\
16 & 1 & 1 & 1 & 1 & 80,3 \\
\hline
\end{tabular}

Tabela 4. Efeitos principais e de interação para as variáveis estudadas na digestão em forno de micro-ondas

\begin{tabular}{cc}
\hline & Efeitos \pm erro padrão \\
\hline Média & $88,6 \pm 1,0$ \\
M & $-5,6 \pm 2,0$ \\
A & $-2,4 \pm 2,0$ \\
S & $-4,9 \pm 2,0$ \\
P & $3,9 \pm 2,0$ \\
M x A & $-6,9 \pm 2,0$ \\
M x S & $0,7 \pm 2,0$ \\
M x P & $0,2 \pm 2,0$ \\
A x S & $2,2 \pm 2,0$ \\
A x P & $-0,6 \pm 2,0$ \\
S x P & $-0,2 \pm 2,0$ \\
M x A x S & $-2,1 \pm 2,0$ \\
M x A x P & $1,5 \pm 2,0$ \\
A x S x P & $-1,1 \pm 2,0$ \\
\hline
\end{tabular}

Intervalo com $95 \%$ de confiança: $\mathrm{t}_{(16,0,95)} \times \mathrm{s}=2,0 \times 2,04=4,04$

Analisando-se a variável ácido sulfúrico, quando se eleva o volume de ácido de 0,2 para 0,6 mL, o efeito médio observado (Tabela 4) é um decréscimo no retorno percentual de 4,9\%. Assim, o nível inferior da variável $\mathrm{S}$ foi selecionado. Embora a potência tenha se mostrado não significativa, o valor do efeito ficou muito próximo ao limite do intervalo com $95 \%$ de confiança $(3,9 \pm 4,0)$. Assim, por medida de segurança optou-se por empregar a potência de $500 \mathrm{~W}$. Como o efeito do tempo de digestão não é significativo para os níveis selecionados, 2 min de digestão foi escolhido. Após esta seleção de níveis para $\mathrm{S}, \mathrm{P}$ e t, chega-se aos experimentos 9 a 12. Destes, a condição mais econômica, para o molibdato e o ácido ascórbico, e com bons resultados no retorno percentual foram nos níveis inferiores para ambos. Portanto, a concentração escolhida de ácido ascórbico foi de $60 \mathrm{mmol} \mathrm{L}^{-1}$ e a concentração de molibdato foi de $16 \mathrm{mmol} \mathrm{L}^{-1}$.

\section{Estudo da adição e recuperação}

Para avaliar o efeito da matriz da amostra na análise do detergente, foi realizado um estudo de adição e recuperação adicionando-se $1 \mathrm{mg}$ $\mathrm{L}^{-1}$ de $\mathrm{P}$. A digestão das amostras de detergente em pó foi realizada com as melhores condições obtidas pelo planejamento fatorial e os resultados são mostrados na Tabela 5.

Tabela 5. Resultados do teste de recuperação para a determinação de fósforo em amostras de detergente em pó usando autoclave

\begin{tabular}{rccc}
\hline Amostras & $\begin{array}{c}\text { Método proposto } \\
\left(\mathrm{mg} \mathrm{g}^{-1}\right)\end{array}$ & $\begin{array}{c}\text { Recuperado } \\
\left(\mathrm{mg} \mathrm{g}^{-1}\right)\end{array}$ & $\begin{array}{c}\text { Recuperação* } \\
(\%)\end{array}$ \\
\hline 1 & $15,1 \pm 0,2$ & $14,4 \pm 0,2$ & 95 \\
2 & $14,6 \pm 0,1$ & $14,4 \pm 0,1$ & 99 \\
3 & $14,6 \pm 0,2$ & $14,8 \pm 0,1$ & 101 \\
4 & $18,2 \pm 0,1$ & $19,5 \pm 0,1$ & 107 \\
5 & $18,5 \pm 0,1$ & $17,1 \pm 0,2$ & 92 \\
\hline
\end{tabular}

$\mathrm{n}=3$. * Amostra contendo $1 \mathrm{mg} \mathrm{L}^{-1} \mathrm{P}$

De acordo com os resultados, observa-se que os valores obtidos no sistema FIA usual ${ }^{26}$ concordaram entre si em nível de confiança de $95 \%\left(\mathrm{t}_{\text {calc }}=1,076\right.$ e $\left.\mathrm{t}_{\text {crítico }}=2,78\right)$ e a recuperação variou entre 92 e $107 \%$. Portanto, não existem problemas de interferência com as amostras de detergente em pó analisadas.

\section{Determinação da concentração de fosfato em amostras de detergentes utilizando detecção espectrofotométrica}

Após estabelecer as melhores condições para os dois tipos de digestão, compararam-se os dois métodos, analisando-se amostras de detergentes em pó.

Os resultados obtidos para a determinação do percentual de $\mathrm{P}$ empregando o sistema FIA, após a digestão das amostras empregando forno de micro-ondas e autoclave, podem ser observados na Tabela 6 .

Tabela 6. Comparação dos resultados obtidos na determinação de P (em \%, $\mathrm{m} / \mathrm{m}$ ) através do preparo da amostra em autoclave e em forno de micro-ondas

\begin{tabular}{ccc}
\hline Amostra & Autoclave & Forno de micro-ondas \\
\hline 1 & $3,57 \pm 0,02$ & $3,32 \pm 0,05$ \\
2 & $0,87 \pm 0,03$ & $0,97 \pm 0,02$ \\
3 & $1,7 \pm 0,1$ & $2,1 \pm 0,1$ \\
4 & $1,52 \pm 0,1$ & $1,70 \pm 0,06$ \\
\hline $\mathrm{n}=3$ & &
\end{tabular}

As digestões das amostras e as determinações de fósforo no sistema FIA foram realizadas em triplicata. De acordo com o teste-t de Student, $\left(\mathrm{t}_{\text {calculado }}=0,788\right.$ e $\left.\mathrm{t}_{\text {tabelado }}=2,78\right)$ não existe diferença significativa entre a digestão com autoclave e forno de micro-ondas em nível de 95\% de confiança. Sendo estáveis os dois tipos de digestão, as amostras podem ser preparadas utilizando-se um forno de microondas, com a vantagem de menor tempo de análise, ou em autoclave, quando houver grande quantidade de amostras a serem digeridas.

Quanto ao teor de fósforo, observa-se que as amostras de detergentes em pó estavam com os teores de $\mathrm{P}$ bem abaixo do limite máximo permitido pelo CONAMA, que é de $4,8 \%$ P por formulação. ${ }^{7}$

\section{CONCLUSÃO}

Os dois procedimentos de abertura de amostra (autoclave e forno de micro-ondas) foram eficientes para as amostras de detergente em pó. Com relação ao tempo gasto para o preparo das amostras, o forno de micro-ondas apresentou menor frequência analítica quando comparado à autoclave, apesar de possuir maior eficiência na transferência da radiação.

O sistema FIA recomendado para determinação de fosfato $\left(\mathrm{PO}_{4}^{3-}\right)$ em amostras de águas foi facilmente adaptado para a análise de deter- 
gente em pó, mantendo suas características iniciais: alta frequência analítica, boa repetibilidade e reduzida geração de resíduos.

As amostras analisadas apresentaram teores de fósforo abaixo do limite máximo estabelecido, ou seja, as diferentes marcas analisadas estão de acordo com a legislação vigente. Entretanto, mesmo que os valores encontrados estejam bem abaixo do que exige a legislação, o ideal seria que fosse utilizado outro tipo de agente estruturante e sequestrante de íons cálcio e magnésio. Alguns detergentes utilizam as zeólitas ${ }^{28}$ para esta função, mas ainda não se sabe qual o efeito destas espécies no meio ambiente.

\section{AGRADECIMENTOS}

Ao Prof. P. Ricardo (IFPE, Ipojuca - PE) e à Agência Estadual de Meio Ambiente e Recursos Hídricos do Estado de Pernambuco (CPRH) pelo forno de micro-ondas e o quarteador das amostras. À Profa. M. F. Pimentel pela valiosa contribuição nos cálculos quimiométricos. Ao CNPq (Proc. 472358/2006-5), à CAPES e PRONEX/ FACEPE/CNPq (Proc. 0333-3.07/06) pelo auxílio financeiro.

\section{REFERÊNCIAS}

1. Carmouze, J. P.; O metabolismo dos ecossistemas aquáticos: fundamentos teóricos, métodos de estudo e análises químicas, $\mathrm{Ed}$. Edgard Blücher Ltda: São Paulo, 1994.

2. Paim, A. P. S.; Pereira, E. R.; Santos, H. R.; Zaiat, M.; Analytica 2004, 12,45 .

3. Souza, J. B.; Daniel, L. A.; Eng. Sanit. Ambient. 2005, 10, 111.

4. Osibanjo, O.; Al-Tamrah, A.; Anal. Chim. Acta 1984, 162, 409.

5. Khanmohammadi, M.; Ashori, A.; Kargosha, K.; Bagheri Garmarudi, A.; J. Surfactants Deterg. 2007, 10, 81.

6. Sostar-Turk, S.; Petrinic, I.; Simonic, M.; Resour. Conserv. Recy. 2005, $44,185$.

7. http:// www.mma.gov.br, acessada em Abril 2010 .

8. Nóbrega, J. A.; Costa, L. M.; Santos, D. M.; Analytica 2002, 1, 32.
9. Fili, S. P.; Oliveira, P. V.; J. Braz. Chem. Soc. 2003, 14, 435.

10. Costa, L. M.; Korn, M. G. A.; Castro, J. T.; Santos, W. P. C.; Carvalho, E. V.; Nogueira, A. R. A.; Quim. Nova 2006, 29, 149.

11. Soylak, M.; Tuzen, M.; Souza, A.S.; Korn, M. G. A.; Ferreira, S. L. C.; J. Hazard. Mater. 2007, 149, 264.

12. Arruda, M. A. Z.; Santelli, R. E.; Quim. Nova 1997, 20, 638.

13. Burguera, J. L.; Burguera, M.; Rondon, C.; Talanta 2002, 58, 1167.

14. Maher, W.; Krikowa, F.; Wruck, D.; Louie, H.; Nguyen, T.; Huang, W. Y.; Anal. Chim. Acta 2002, 463, 283.

15. Dallago, R. M.; Delanora, R.; Dressler, V. L.; Flores, E. M. M.; Resumos da $29^{a}$ Reunião Anual da Sociedade Brasileira de Química, Águas de Lindóia, Brasil, 2006.

16. Buanuam, J.; Miro, M.; Hansen, E. H.; Shiowatana, J.; Estela, J. M.; Cerdá, V.; Talanta 2007, 71, 1710.

17. Longman, G. F.; The analysis of detergents and detergent products, John Wiley \& Sons: New York, 1975.

18. Grassi, V.; Dias, A. C. B.; Zagatto, E. A. G.; Talanta 2004, 64, 1114

19. Ruzicka, J.; Hansen, E. H.; Anal. Chim. Acta 1975, 78, 145.

20. Ruzicka, J.; Marshall, G.; Anal. Chim. Acta 1990, 237, 329.

21. Reis, B. F.; Giné, M. F.; Zagatto, E. A. G.; Lima, J. L. F. C.; Lapa, R. A.; Anal. Chim. Acta 1994, 293, 129.

22. Jing-Fu, L.; Gui-Bin, J.; Talanta 2000, 52, 211.

23. Barros Neto, B.; Scarminio, I. S.; Bruns, R. E.; Quim. Nova 2006, 29, 1401.

24. Barros Neto, B.; Scarminio, I. S.; Bruns, R. E.; Planejamento e Otimização de Experimentos, Ed. Unicamp: Campinas, 1996.

25. Pereira-Filho, E. R.; Poppi, R. J.; Arruda, M. A. Z.; Quim. Nova 2002, 25, 246.

26. Karlberg, B.; Pacey, G. E.; Flow Injection Analysis: A pratical guide, Elsevier: Amsterdam, 1989.

27. Standard Methods for the Examination of Water and Wastewater, American Public Health Association, American Water Works Association, Water Pollution Control Federation: Washington, D.C., $19^{\text {th }}$ ed., 1998.

28. Osório, V. K. L.; de Oliveira, W.; Quim. Nova 2001, 24, 700. 